\title{
Evaluation of the Association between Lower Urinary Tract Symptoms and Erectile Dysfunction, Considering its Multiple Risk Factors
}

\author{
Ernani Luis Rhoden, MD, PhD, ${ }^{*}$ Charles Edison Riedner, $\mathrm{MD},{ }^{\dagger}$ Alexandre Fornari, MD, ${ }^{\ddagger}$ \\ Sandra Costa Fuchs, MD, PhD, ${ }^{\S}$ and Eduardo Porto Ribeiro, MDף \\ *Surgery Department, Urology Division, at UCSPA and Postgraduate Course in Medical Sciences at UFRGS, Porto \\ Alegre, Brazil; ${ }^{\dagger}$ Postgraduate Course in Medical Sciences at UFRGS and Urology Service of Hospital Geral of \\ Porto Alegre, Brazilian Army Hospital, Porto Alegre, Brazil; łPostgraduate Course in Medical Sciences at UFRGS, Porto \\ Alegre, Brazil; §Postgraduate Course in Medical Sciences at UFRGS and Ultralitho Centro Médico, Florianópolis, Brazil; \\ "Social Medicine Department at The UFRGS School of Medicine, Porto Alegre, Brazil
}

DOI: $10.1111 / \mathrm{j} .1743-6109.2008 .00877 . x$

\section{A B S T R A C T}

Aim. To investigate the relationship between lower urinary tract symptoms (LUTS) and erectile dysfunction (ED), while considering multiple risk factors for ED, including an anthropometric evaluation of central obesity.

Methods. A cross-sectional study was carried out with 192 consecutive male subjects ( $\geq 40$ years old). Conditions clearly associated with ED, other than obesity and age, were considered exclusion criteria. Men were evaluated routinely for clinical history, received a physical examination, and were subjected to blood analysis for fasting serum glucose, lipid profile, and serum testosterone. Patients with previous known history of diabetes mellitus or hypertension were excluded. Anthropometric measures taken included body mass index (general obesity) and waist circumference, waist-hip index, and sagittal abdominal diameter (visceral obesity). Analyses were performed using bivariate and multivariate models (multiple logistic regression). Age, education, alcohol consumption, smoking, sedentary lifestyle, fasting blood glucose level, dyslipidemia, hypogonadism, general obesity, and visceral obesity were taken into account as potential confounding factors.

Main Outcome Measures. All men completed the International Index of Erectile Function and International Prostate Symptom Score (IPSS).

Results. IPSS scores were low, intermediate, and high in 89 (46.4\%), 76 (39.6\%), and 27 (14.1\%) men, respectively. Overall IPSS scores were significantly associated with ED $(P=0.002)$. In addition, an association between the severity of ED and LUTS was observed $(P=0.008)$. The mean quality of life assessment in the IPSS revealed a statistically significant difference between individuals with varying degrees of $\mathrm{ED}(P=0.008)$. The logistic regression analyses showed that IPSS scores and ED remained independently associated even after the control for confounding factors (odds ratio $=1.07,95 \% \mathrm{CI}=1.02-1.13, P=0.01$ ).

Conclusion. This study suggests that LUTS are independently associated with ED, taking into account various risk factors for ED, including visceral obesity. Rhoden EL, Riedner CE, Fornari A, Fuchs SC, and Ribeiro EP. Evaluation of the association between lower urinary tract symptoms and erectile dysfunction, considering its multiple risk factors. J Sex Med 2008;5:2662-2668.

Key Words. Erectile Dysfunction; Obesity; Sexual Dysfunction; Lower Urinary Tract Symptoms; LUTS

\section{Introduction}

$\mathrm{L}$ ower urinary tract symptoms (LUTS) and erectile dysfunction (ED) are common urologic conditions that are seen in the aging male, with estimated prevalences of $56 \%$ and $40 \%$, respectively, in men $>40$ years old [1]. The high prevalence of these conditions may explain the coincidence of LUTS and ED in older men: in one study [2], $77 \%$ of men diagnosed with ED 
presented with LUTS. Until recently, little evidence was available to support a consistent relationship between LUTS and ED. Recent epidemiological studies have demonstrated that these two clinical conditions are independently related, despite the effect of other recognized risk factors for both conditions, such as age, systemic hypertension, dyslipidemia, cardiovascular diseases, and lifestyle factors $[3,4]$. Thus, LUTS is now considered an important risk factor for sexual dysfunction [5], and a potential common cause has been suggested [6].

Recently, various theories have been proposed regarding the common pathophysiology between LUTS and ED, including autonomic hyperactivity, the presence of pelvic atherosclerosis, reduced production of nitric oxide in the pelvic organs including the prostate and penis, increased RHOkinase activation and endothelin activity, and influences of a metabolic syndrome which affects both voiding and erectile function. However, a mechanistic association between ED and LUTS has not been definitively established [2,3,5-10].

To determine the relationship between LUTS and ED, a careful epidemiological analysis, including other risk factors for ED, must be conducted. Previous work has only considered the contribution of general obesity, measured by body mass index [9]. However, a recent report suggested a strong independent association between central obesity and ED [11].

\section{Aims}

In the present study we have investigated the association between LUTS and ED, while considering the multiple risk factors for ED, including an anthropometric evaluation of central obesity.

\section{Methods}

Between August 2003 and July 2004, a total of 1,981 patients were seen at a large state outpatient clinic for primary health care and urologic center in the city of Porto Alegre, southern Brazil. From this population, all men aged $\geq 40$ years (total $=623$ ) were eligible for inclusion, regardless of their reason for consultation. Exclusion criteria included previous diagnosis of diabetes mellitus $(17.66 \%)$, systemic arterial hypertension $(15.25 \%)$, previous radiotherapy $(1.61 \%)$ or chemotherapy $(1.44 \%)$, pelvic or orthopedic surgery $(3.53 \%)$, heart or peripheral vascular diseases $(10.43 \%)$, psychiatric disease $(2.41 \%)$, use of psy- chotropic medications, anxiolytic agents, or mood stabilizers (11.24\%), current or previous treatment for $\mathrm{ED}(5.14 \%)$, or use of medications that may affect the levels or action of androgens $(0.48 \%)$. In total, 192 men were included in the study. The study was approved by our institution's Committee on Ethics in Research.

Erectile function was evaluated by the International Index of Erectile Function (IIEF), with the questionnaire being completed by the subject or, if required, by a certified interviewer. Answers to questions $1-5$ and 15 provided the score for the erectile domain, and the cases were classified as without ED (score $>25$ points) or with any intensity of $\mathrm{ED}$ (score $\leq 25$ points). Cases were also classified according to intensity: mild ED (score: 17-25), moderate ED (score $=11-16$ ), and severe ED (score $\leq 10$ points).

LUTS were evaluated with the International Prostate Symptom Score (IPSS). The sum of the score for the first seven questions allowed to classify the subjects into the low score range (0-7), intermediate score range (8-19), and high score range (20-35). The eighth question, regarding the subject's quality of life (QoL), was considered separately, with a score range from 1 to 6 .

A single investigator performed the standardized anthropometry in triplicate, and was blinded to the IIEF and IPSS outcomes. Four variables were selected, one for general obesity (body mass index) and three for visceral obesity (waist circumference, waist-hip index, and sagittal abdominal diameter). Body mass index was calculated as the ratio between weight $(\mathrm{kg})$ and the height squared $\left(\mathrm{m}^{2}\right)$. Normal values ranged from $20 \mathrm{~kg} / \mathrm{m}^{2}$ to $24.9 \mathrm{~kg} / \mathrm{m}^{2}$, overweight values from $25.0 \mathrm{~kg} / \mathrm{m}^{2}$ to $29.9 \mathrm{~kg} / \mathrm{m}^{2}$, and obese values were $\geq 30 \mathrm{~kg} / \mathrm{m}^{2}$. Circumference values were obtained from the perpendicular plane along the body axis, in scales of $0.5 \mathrm{~cm}$. The waist circumference measurement was obtained from the midpoint between the iliac crest and the costal margin; values greater than the 85 th percentile $(102 \mathrm{~cm})$ were considered central obesity. The waist-hip index was calculated using the ratio between the waist and hip (taken at the level of the femoral trochanters) circumferences, with the 10th percentile (0.91) as a cut-off for central obesity. Sagittal abdominal diameter was assessed in dorsal decubitus position, using the distance from the exam table to the highest point of the subject's abdomen. Sagittal diameter $>24.5 \mathrm{~cm}$ (the 75 th percentile) was also used as cut-off for central obesity. 
During the same interview, confounding factors associated with ED were investigated, including age [12], formal education $<8$ years of school, smoking [12,13], sedentary lifestyle [12-14] $(<120$ minutes/week of physical activity, independent of the intensity [12]), and abusive consumption of alcoholic beverages [13] ( $\geq 350 \mathrm{~g} /$ week of ethanol or a daily consumption of at least 3.5 glasses of wine, two bottles of beer, or two doses of spirits [12]).

A fasting blood sample was obtained from all subjects, and glucose, total cholesterol, highdensity lipoprotein (HDL), low-density lipoprotein (LDL), triglycerides, and total testosterone (radioimmunoassay, normal range: 300-1,000 ng/ $\mathrm{dL}$ ) were determined, all in the same laboratory. The presence of dyslipidemia was defined by serum levels of total cholesterol $>240 \mathrm{mg} / \mathrm{dL}$, $\mathrm{LDL}>130 \mathrm{mg} / \mathrm{dL}, \quad$ triglycerides $>150 \mathrm{mg} / \mathrm{dL}$ and/or $\mathrm{HDL} \leq 40 \mathrm{mg} / \mathrm{dL}$, and hypogonadism as serum levels of total testosterone $<300 \mathrm{ng} / \mathrm{dL}$. The presence of dyslipidemia [14], hypogonadism [15], and fasting blood glucose [14] were also considered potential confounding factors.

The presence and degree of LUTS were analyzed and correlated with the presence and degree of ED, with statistical significance assessed by Pearson's Chi-square using SPSS ${ }^{\circledR}$ v.12 software (SPPS Inc., Chicago, IL, USA). Student's $t$-test or the Wilcoxon-Mann-Whitney test were used as appropriate for means or ranks comparison. $P$ values $<0.05$ were considered statistically significant. The QoL means were analyzed with the three-degree of IPSS as covariates, using one-way ANOva with Tukey post hoc analysis. The IPSS scores (taken as a continuous variable) and QoL scores were analyzed for correlation with IIEF, using Pearson's coefficient.

Associations between the LUTS and ED were tested using logistic regression, adjusted for history and laboratory confounding factors (including age, education, sedentary lifestyle, smoking, abusive consumption of alcohol, fasting blood glucose, dyslipidemia, and hypogonadism) and for anthropometry: body mass index (general obesity) and waist circumference, waist-hip index, and sagittal abdominal diameter (visceral obesity). Crude and adjusted odds ratios and 95\% confidence intervals were obtained for LUTS as a predictor of $\mathrm{ED}$ (IIEF $\leq 25$ points). This model was initially tested in the entire subject population using IPSS as a continuous variable. Thereafter, the same model was tested by including the IPSS score as a categorical variable, with the $2 \mathrm{nd}, 3 \mathrm{rd}$, and 4th quartiles compared to the 1st, in order to determine whether the last three quartiles odds ratio serve as predictors of ED.

\section{Results}

All 192 recruited subjects agreed to participate in the study. The demographic, socioeconomic, behavioral, and clinical characteristics for all subjects are presented in Table 1. The participants' ages ranged from 40 to 81 years (mean = $57.8 \pm 9.1$ years); $2.6 \%$ were illiterate, $67.2 \%$ attended school for $<8$ years, $19.3 \%$ for $9-11$ years, and $11 \%$ for $>11$ years; $15.1 \%$ exhibited excessive consumption of alcoholic beverages; $69.8 \%$ were sedentary and $17.7 \%$ were smokers.

Table 1 Demographic, socioeconomic, behavioral, and clinical characteristics of the study population-overall and stratified by the presence of ED ( $\mathrm{N}$ and \%, mean \pm standard deviation or (median [25\%-75\%])

\begin{tabular}{lll}
\hline Characteristics & Total & Men with ED (IIEF < 25) N = 147 \\
\hline White skin color & $159(82.8 \%)$ & $123(83.7 \%)$ \\
Education (8 years or less at school) & $155(80.7 \%)$ & $117(79.6 \%)$ \\
Smoking & $34(17.7 \%)$ & $28(19 \%)$ \\
Sedentary life style & $134(69.8 \%)$ & $98(66.7 \%)$ \\
Alcohol consumption & $29(15.1 \%)$ & $24(16.3 \%)$ \\
Total testosterone $(\mathrm{ng} / \mathrm{dL})$ & $472.7(381-614.8)$ & $481.8(378-620)$ \\
Triglycerides $(\mathrm{mg} / \mathrm{dL})$ & $118(87.2-160.5)$ & $117(82.0-161.0)$ \\
Total cholesterol $(\mathrm{mg} / \mathrm{dL})$ & $203.6 \pm 40.5$ & $202.2 \pm 39.5$ \\
HDL-cholesterol $(\mathrm{mg} / \mathrm{dL})$ & $44.6 \pm 11.1$ & $44.3 \pm 11.1$ \\
LDL-cholesterol $(\mathrm{mg} / \mathrm{dL})$ & $130.7 \pm 34.6$ & $130.7 \pm 34.1$ \\
Fasting blood glucose $(\mathrm{mg} / \mathrm{dL})$ & $94(84.0-106.8)$ & $95(84.0-111.0)$ \\
Waist circumference $(\mathrm{cm})$ & $92.9 \pm 9.2$ & $92.4 \pm 9.3$ \\
Sagittal abdominal diameter & $22.7 \pm 3.7$ & $22.6 \pm 3.8$ \\
Waist-hip index & $0.95 \pm 0.03$ & $0.95 \pm 0.38$ \\
Body mass index $\geq 30 \mathrm{~kg} / \mathrm{m}^{2}$ & $46(24.0 \%)$ & $63(42.9 \%)$ \\
Body mass index $25-29.9 \mathrm{~kg} / \mathrm{m}^{2}$ & $87(45.3 \%)$ & $35(23.8 \%)$ \\
\hline
\end{tabular}

$\mathrm{ED}=$ erectile dysfunction; IIEF = International Index of Erectile Function; HDL = high-density lipoprotein; LDL = low-density lipoprotein. 
Table 2 Presence and degree of erectile dysfunction according to the International Prostate Symptom Score intensity ( $\mathrm{N}$ and \%)

\begin{tabular}{llllrrr}
\hline \multicolumn{7}{c}{ Erectile dysfunction } \\
\cline { 2 - 7 } & No (IIEF > 25) & Mild (IIEF 17-25) & Moderate (IIEF 11-16) & Severe (IIEF < 11) & Total \\
\hline International & $0-7$ & $31(34.8 \%)$ & $33(37.1 \%)$ & $9(10.1 \%)$ & $16(18 \%)$ & 89 \\
Prostate Symptom & $8-19$ & $10(13.2 \%)$ & $31(40.8 \%)$ & $21(27.6 \%)$ & $14(18.4 \%)$ & 76 \\
Score & $>19$ & $4(14.8 \%)$ & $9(33.3 \%)$ & $7(25.9 \%)$ & $7(25.9 \%)$ & 27 \\
Total & $45(23.4 \%)$ & $73(38 \%)$ & $37(19.3 \%)$ & $37(19.3 \%)$ & 192 &
\end{tabular}

Pearson Chi-squared: $17.52 ; P=0.008$ (df: 6 ).

IIEF = International Index of Erectile Function.

Overall prevalence of ED was $76.6 \%$, with a mean of IIEF score of $18.5 \pm 7.8$.

In the study population, the reported IPSS scores were in the lower range ( $0-7$ points) in 89 ( $46.4 \%)$ subjects, intermediate range ( $8-19$ points) in $76(39.6 \%)$ subjects, and in the high range (20-35 points) in 27 (14.1\%) subjects. An analysis of IPSS quartiles indicated that scores of 3,8 , and 15 fell into the 25 th, 50 th, and 75 th percentiles, respectively.

The association between LUTS and ED is shown in Table 2. In men with mild symptoms of LUTS, the prevalence of ED was $65.2 \%$ (58 of 89 men), but when moderate and severe symptoms of LUTS were considered, the rates of ED were $86.8 \%$ (66 of $76 \mathrm{men}$ ) and $85.2 \%$ ( 23 of $27 \mathrm{men}$ ), respectively $(P=0.002)$. In addition, a significant association between the severity of ED and LUTS was observed (Table 2). It is interesting to note that in men with normal erectile function (IIEF $>25$ ) the occurrence of mild urinary symptoms were more common $(68.9 \%)$ than moderate $(22.2 \%)$ or severe $(8.9 \%)$ symptoms. Thus, among men with scores of the IIEF in the ED range $(\leq 25$ points) there is a tendency toward an increased prevalence of moderate and severe LUTS, with a decreased frequency of mild urinary symptoms. This was also suggested too by the significant negative correlation between IIEF and IPSS $(r=-0.23, P=0.01)$.

The QoL measurements revealed a significant difference between the various degrees of ED $(P=0.008)$, with post hoc analysis indicating that moderate ED was significantly different in QoL scores than both mild ED and no ED $(P=0.04$ and $P=0.007$, respectively, Figure 1). The negative correlation between these two variables is significant $(r=-0.21, P=0.003)$.

After controlling for confounding variables, including central obesity, a significant and independent association between urinary symptoms (IPSS) and ED was observed $(P=0.01$; Table 3$)$.
We noted that, in men in the $3 \mathrm{rd}$ and 4th quartiles for IPSS scores, relative to the 1st quartile the calculated odds ratio for ED was $3.45(95 \%$ $\mathrm{CI}=1.22-9.77)$ and $3.76(95 \% \mathrm{CI}=1.29-11.00)$, respectively (Table 3 ). Based on these analyses, the IPSS scores equal to or above eight represented a significant increase in the risk (odds ratio) for the presence of ED in this study population.

\section{Discussion}

The present study identified an independent association between LUTS and ED as well as a significant association between the intensity of both conditions. We also observed an independent effect of IPSS in predicting ED, regardless of the adjustment for confounding factors such as age,

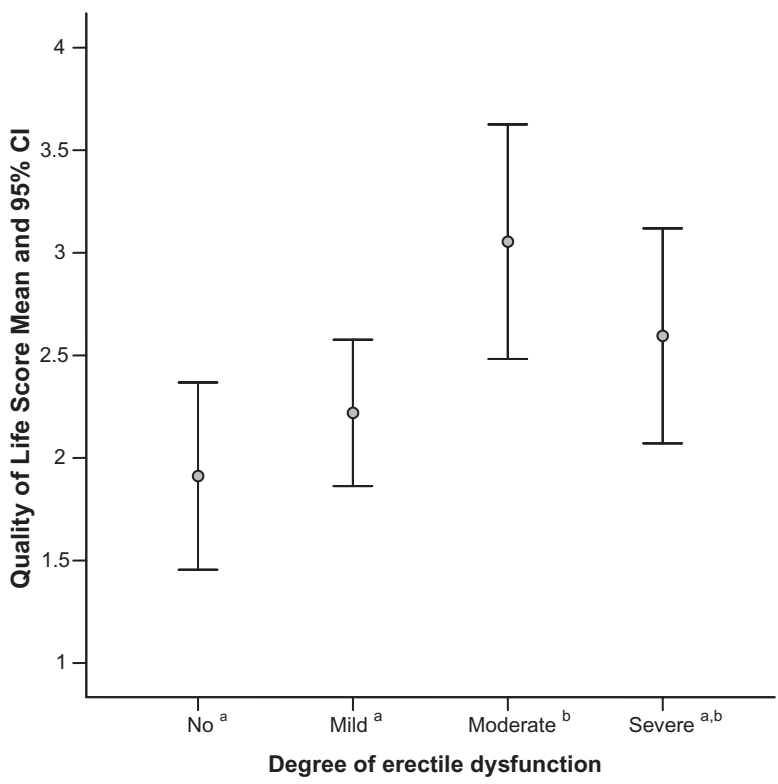

Figure 1 Quality of life assessment from the International Prostate Symptom Score, considering the presence and degree of erectile dysfunction. ANOVA $F$ : 4.08 , df: 3 , $P=0.008$. a, b: Post hoc analysis (Tukey), $P<0.05$. 
Table 3 Effect of IPSS as a predictor of erectile dysfunction, with total score range and IPSS quartile (reference: 1st quartile)

\begin{tabular}{llll}
\hline & $\beta$ Coefficient & $\beta$ Standard error & $\begin{array}{l}\text { Adjusted odds ratio } \\
(95 \% \mathrm{Cl})^{\star}\end{array}$ \\
\hline $\begin{array}{l}\text { All score range } \\
\text { IPSS quartile }\end{array}$ & 0.07 & 0.03 & $1.07(1.02-1.13)^{\dagger}$ \\
Score 3-8 (2nd quartile) & 0.37 & 0.48 & $1.44(0.56-3.70)$ \\
Score 8-15 (3rd quartile) & 1.24 & 0.53 & $3.45(1.22-9.77)$ \\
Score $>$ 15 (4th quartile) & 1.33 & 0.55 & $3.76(1.29-11.00)$ \\
\hline
\end{tabular}

${ }^{\star} P=0.01$.

†Odds ratio adjusted for age, education, excessive consumption of alcoholic beverages, smoking, sedentary lifestyle, fasting blood glucose, dyslipidemia, hypogonadism, general obesity (body mass index), and visceral obesity (waist-hip index, sagittal abdominal diameter, and waist circumpherence).

IPSS = International Prostate Symptom Score.

education, excessive consumption of alcoholic beverages, smoking, sedentary lifestyle, fasting blood glucose, dyslipidemia, hypogonadism, general obesity (body mass index), and visceral obesity (waist-hip index, sagittal abdominal diameter, and waist circumference). Moreover, the odds ratios for $\mathrm{ED}$ were 3.45 (95\% $\mathrm{CI}=1.22-9.77)$ and $3.76(95 \% \mathrm{CI}=1.29-11.00)$ for men in the $3 \mathrm{rd}$ and 4th quartiles of IPSS, respectively, relative to the 1 st quartile. To the best of our knowledge, this is a novelty in the assessment of the association between LUTS and ED and establishes visceral obesity as a meaningful confounding factor in this relationship.

The prevalence of LUTS varies internationally [16], and is reported to occur in $45 \%$ of men in Sweden [8] and 75\% in Egypt [2]. Specifically, Glina et al. [1] reported a prevalence of $56 \%$ of moderate and severe LUTS scores in men 40 years old or older. Despite this large country-to-country variation, a significant increase in LUTS with age has been reported by several authors [16].

Nevertheless, the high prevalence of ED and LUTS in older men has stimulated research concerning their comorbidity, because both conditions significantly reduce quality of life reports [6]. Aslan et al. [4] reported a significant negative correlation between IIEF and IPSS scores, after controlling for confounding factors such as age and other known ED risk factors. Similarly, numerous others authors $[1,4,5,9]$ reported a strong independent association between LUTS and ED. In particular, a correlation between the severity of LUTS and ED has been described $[2,9,10]$. For example, Shiri et al. [9] reported an adjusted odd ratio for ED of 2.6 when IPSS scores were between 11-19 points and 4.4 for IPSS scores $\geq 20$ points. The adjusted odds ratio calculated from the present data are consistent with the results of Shiri and colleagues, since an odds ratio greater than three was demonstrated for IPSS scores in the 3rd
(8-15 points) and 4th (scores $>15)$ quartiles relative to the 1st (1-3 points) quartile, indicative of a severity relationship of IPSS and ED.

This statistically significant association, described in the literature, has led to four hypotheses concerning the possible common pathophysiology of LUTS and ED. The theory of autonomic hyperactivity highlights the effects of a metabolic syndrome and its components, such as hypertension, obesity, and hyperinsulinemia, which are associated with autonomic activation. According to this theory, these alterations give rise to ED and also effects in the bladder and urethra, which underlie LUTS pathogenesis $[3,6,7]$. The nitric oxide theory is based on the finding that conditions such as aging, hypertension, smoking, hypercholesterolaemia, and diabetes, which are also risk factors for ED, can decrease the release of nitric oxide in the pelvic organs, including the prostate and penis. This in turn increases smooth muscle cells' contractile forces at the corpus cavernosum, bladder neck, and prostate urethra, resulting in an increase of the bladder outlet resistance as well as venous leakage from the penis associated with poor smooth muscle relaxation $[3,6,7]$. The theory of increased Rho-kinase activation and endothelin activity has been suggested by experimental studies, which have demonstrated an increase in Rho-kinase activity (and consequently increased calcium sensitivity of the contractile machinery) in the detrusor as well as in the corpus cavernosum of rabbits with partial bladder outlet obstruction. Since the actions of several factors aside from norepinephrine (e.g., endothelin-1 and angiotensin II), which may be involved in the increased smooth muscle activity in both LUTS and ED, are dependent on Rho-kinase activity that acts downstream from these receptors, it has been suggested that the common link between these two conditions is increased Rho-kinase 
activity [7]. The fourth theory suggests a common vascular pathway, since low peak systolic velocity of the cavernosal arteries and poor response to intracorporeal injection were observed in individuals with LUTS and arteriogenic and neurogenic ED [17].

These theories of common causality have been contested in the literature $[6,7,18]$, and some authors have argued against the presence of an established basis of causality, despite the everincreasing number of epidemiological studies. In this respect, Stromberg et al. [8] cited a significant but weak association between ED and LUTS (correlation coefficient of $-0.29, P<0.001)$ and recommended further studies to better understand the possible causal relationship. This weak effect is consistent with the present findings of a low odds ratio of 1.07 (95\% CI $=1.02-1.13)$ for $\mathrm{ED}$ when all IPSS values were considered (continuous variable). Similarly, the correlation coefficients detected for IPSS or QoL and IIEF $(r=-0.23$ and $r=-0.21$, respectively), although significant, are weak. Thus, in our analysis, a significant relationship could be observed, but this effect is diminished by the low magnitude of the effect. However, these finding added strength to a common causality theory for both conditions.

Previous analyses of the relationship between LUTS and ED were controlled for factors known to be associated with ED, such as smoking $[12,13]$, education [19], excessive consumption of alcohol [13], hyperglycemia [14], dyslipidemia $[14,20]$, hypogonadism, [15], and sedentary lifestyle [12-14]. In all these studies, only general obesity was considered and controlled as confounding factor. The importance of central obesity, evaluated by anthropometry, in predicting ED has been recently reviewed by Rhoden et al. [11]. In this study, an independent association between ED and central obesity was demonstrated, despite controlling for the effects of various confounding factors, including general obesity (body mass index). This result highlights the importance of obesity assessment due to the age-related redistribution of body fat. The evaluation of central obesity is mandatory when evaluating confounding factors that may interact in LUTS and ED.

Some limitations of the present study could be attributed to the population size and the source of participants' recruitment-a urologic center-that explains the high prevalence of ED. However, the test of hypothesis required such selection of participants in order to assess the association between these conditions. The alternative-to select men from a general population-would require a very large sample size and it might be less cost-effective. On the other hand, the aim of the article was not to provide prevalence rate of $\mathrm{ED}$ for a general population. In addition, the rigid eligibility criteria allowed excluding participants with diabetes and hypertension and the measurements were conducted in an appropriate environment. Finally, the multivariate analyses adjusting for confounding factors strengthened the role of central obesity as the novelty of this study.

\section{Conclusion}

In conclusion, the data from the present study showed that ED and LUTS are associated clinical conditions and that LUTS is independently associated with ED even when confounding risk factors for the latter condition are taken into account, including visceral obesity. The possibility of a common causal relationship between LUTS and ED requires further epidemiological investigation.

\section{Acknowledgment}

Prof. Rhoden is researcher of Conselho Nacional de Desenvolvimento Científico e Tecnológico $\left(\mathrm{CNP}_{9}\right)$ "National Counsel of Technological and Scientific Development"-Brazil.

Corresponding Author: Ernani Luis Rhoden, MD, $\mathrm{PhD}$, Surgery Department, Urology Division, at UCSPA and Postgraduate Course in Medical Sciences at UFRGS, Rua Jaragua 370/302 Porto Alegre, RS-Brazil 90450-140. Tel: 555133333144 ; Fax: 5551 33333144; E-mail: ernanirhoden@yahoo.com.br

Conflict of Interest: None declared.

\section{Statement of Authorship}

\section{Category 1}

(a) Conception and Design

Ernani Luis Rhoden; Charles Edison Riedner; Alexandre Fornari; Eduardo Porto Ribeiro; Sandra Costa Fuchs

(b) Acquisition of Data

Ernani Luis Rhoden; Charles Edison Riedner; Alexandre Fornari; Eduardo Porto Ribeiro; Sandra Costa Fuchs

(c) Analysis and Interpretation of Data

Ernani Luis Rhoden; Charles Edison Riedner; Alexandre Fornari; Eduardo Porto Ribeiro; Sandra Costa Fuchs 


\section{Category 2}

(a) Drafting the Article

Ernani Luis Rhoden; Charles Edison Riedner; Alexandre Fornari; Eduardo Porto Ribeiro; Sandra Costa Fuchs

(b) Revising It for Intellectual Content

Ernani Luis Rhoden; Charles Edison Riedner; Alexandre Fornari; Eduardo Porto Ribeiro; Sandra Costa Fuchs

\section{Category 3}

(a) Final Approval of the Completed Article

Ernani Luis Rhoden; Charles Edison Riedner; Alexandre Fornari; Eduardo Porto Ribeiro; Sandra Costa Fuchs

\section{References}

1 Glina S, Santana AW, Azank F, Mello LF, Moreira ED, Jr. Lower urinary tract symptoms and erectile dysfunction are highly prevalent in ageing men. BJU Int 2006;97:763.

2 El-Sakka AI. Lower urinary tract symptoms in patients with erectile dysfunction: Analysis of risk factors. J Sex Med 2006;3:144.

3 Rosen RC. Update on the relationship between sexual dysfunction and lower urinary tract symptoms/benign prostatic hyperplasia. Curr Opin Urol 2006;16:11.

4 Aslan G, Cavus E, Karas H, Oner O, Duran F, Esen A. Association between lower urinary tract symptoms and erectile dysfunction. Arch Androl 2006; $52: 155$.

5 Rosen RC. Assessment of sexual dysfunction in patients with benign prostatic hyperplasia. BJU Int 2006;97(2 suppl):29.

6 McVary KT. Interrelation of erectile dysfunction and lower urinary tract symptoms. Drugs Today (Barc) 2005;41:527.

7 McVary K. Lower urinary tract symptoms and sexual dysfunction: Epidemiology and pathophysiology. BJU Int 2006;97(2 suppl):23.

8 Stroberg P, Boman H, Gellerstedt M, Hedelin H. Relationships between lower urinary tract symptoms, the bother they induce and erectile dysfunction. Scand J Urol Nephrol 2006;40:307.

9 Shiri R, Hakkinen J, Koskimaki J, Huntala H, Auvinen A, Hakama M, Tammela TL. Association between the bothersomeness of lower urinary tract symptoms and the prevalence of erectile dysfunction. J Sex Med 2005;2:438.
10 Yassin A, Saad F, Hoesl CE, Traisch AM, Hammadeh M, Shabsigh R. Alpha-adrenoceptors are a common denominator in the pathophysiology of erectile function and BPH/LUTS-implications for clinical practice. Andrologia 2006;38:1.

11 Riedner CE, Rhoden EL, Ribeiro EP, Fuchs SC. Central obesity is an independent predictor of erectile dysfunction in older men. J Urol 2006;176: 1519.

12 Feldman HA, Johannes CB, Derby CA, Kleinman KP, Mohr BA, Araujo AB, McKinlay JB. Erectile dysfunction and coronary risk factors: Prospective results from the Massachusetts male aging study. Prev Med 2000;30:328.

13 Bacon CG, Mittleman MA, Kawachi I, Giovannucci E, Glasser DB, Rimm EB. Sexual function in men older than 50 years of age: Results from the health professionals follow-up study. Ann Intern Med 2003;139:161.

14 Ponholzer A, Temml C, Mock K, Marszalek M, Obermayr R, Madersbacher S. Prevalence and risk factors for erectile dysfunction in 2,869 men using a validated questionnaire. Eur Urol 2005;47:80.

15 Ahn HS, Park CM, Lee SW. The clinical relevance of sex hormone levels and sexual activity in the ageing male. BJU Int 2002;89:526.

16 Fourcade RO. [The UrEpik study: A descriptive epidemiological approach to lower urinary tract symptoms, sexual disorders and urinary continence in four countries]. Ann Urol (Paris) 2005;39(5 suppl):S139.

17 El-Sakka AI. Lower urinary tract symptoms in patients with erectile dysfunction: Is there a vascular association? Eur Urol 2005;48:319.

18 Costabile RA, Steers WD. How can we best characterize the relationship between erectile dysfunction and benign prostatic hyperplasia? J Sex Med 2006;3:676.

19 Mirone V, Ricci E, Gentile V, Basile Fasolo C, Parazzini F. Determinants of erectile dysfunction risk in a large series of Italian men attending andrology clinics. Eur Urol 2004;45:87.

20 Rosen RC, Fisher WA, Eardley I, Niederberger C, Nadel A, Sand M. The multinational Men's Attitudes to Life Events and Sexuality (MALES) study: I. Prevalence of erectile dysfunction and related health concerns in the general population. Curr Med Res Opin 2004;20:607. 